\title{
An Introduction of Face Recognition and Face Detection for Blurred and Noisy Images
}

\author{
Ratnesh Kumar Shukla $^{1 *}$, Ajay Agarwal ${ }^{2}$, Anil Kumar Malviya ${ }^{3}$ \\ ${ }^{1}$ Department of Computer Science \& Engineering, Dr.AKTU Lucknow, Uttar Pradesh, India \\ ${ }^{2}$ Department of Computer Science \& Engineering, KIET Ghaziabad, Uttar Pradesh, India \\ ${ }^{3}$ Department of Computer Science \& Engineering, KNIT Sultanpur Uttar Pradesh, India \\ Available online at: www.isroset.org
}

Received: 03/Jun/2018, Revised: 09/Jun/2018, Accepted: 17/Jun/2018, Online: 30/Jun/ 2018

\begin{abstract}
In this paper the proposed model is using for the identity to the noisy and blurred images. In our surrounding they are a big problem of randomly change the environment, or climate change. Image processing, are working in different platform. Such as Pattern Recognition, Computer vision Pattern, Machine Learning and Artificial Intelligence is using for the authentication of unauthorised person. Images are using for the authentication and verification. Authentication and Registration is the initial step of the identification and verification of the object. In this paper we are introducing blur and noisy images. And compare these images in our database. If images are verified from proposed model then they registered in the database for future use. The problem is obtaining in unclear images. Blur and Noise is the main disturbance of the images found in captured process. This problem obtain when we are capturing the images. They are found in the presence of dust and lighting. So in this technique we are remove the noise and blur of images. In this proposed model for deblur and denoise is work on corrupted images. In current scenario there are different algorithms working on the quality of images. Images are in pixel from and there are found in million colours in images. Then it is found very difficult to original images. When we are using high quality of cameras then it is possible to capture good quality images. But when we are using these novel method, we are if recognize the image. So we are using proposed algorithm to identify the images. We are comparing the trainee images to store our database. Then we found a real image and registered in the database.
\end{abstract}

Keywords: Blurred and Noise Image, Wiener Filter, RANSAC algorithm, Face Recognition System, Templates, Data storage.

\section{INTRODUCTION}

Face detection and Face recognition is very important task to identification for Authentication and Verification. There is very difficult task to identify the faces from noisy and blurred images. When, we are working on the blurred and noisy faces. They are not reacting as a particular object. It is important how to access the faces. First of all there is some step how to capture the faces. In figure 1 there are some steps involved to flow of captured faces [1].

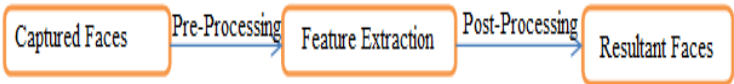

Figure l: Processing Flow Of Capured Faces

Face detection and recognition is a process of images. Face recognition system is very powerful for low-cost desktop face detector [2]. In real time computing

Systems and Face recognition system are working a good job in automatic face processing in digital images. After comparing applications we found different variety of images. Such as Biometric Authentication, Surveillance for military services, Bioinformatics, Pattern Analysis, and Multimedia applications. Researcher and developer are working in automatic face recognition system for noisy and blurred images. Captured faces are the first step in automated face recognition. Its reliability and availability has a major influence on the performance and liability of the complete face recognition system [3]. A captured faces should be able to identify and locate the entire present facial feature regardless of their resolution, angle, pose, colour, scale, orientation, expression and age [4].

\section{Processing Flow of Captured Faces}

This is very difficult process, how to capture the faces? In this process we are using automatic face detector digital camera [5]. Face detector digital camera is focused on the faces of the images. Digital camera is focus on the faces from images. Face detector digital camera is capturing the complete image but the assign only the faces. Because these are include the feature of the faces [6]. With the help of this algorithm, we identified the images in true configuration such that pose, brightness and contrast etc. Then we will go to next step, which are called pre-processing.

Pre-processing is the second step of processing flow of captured faces. When we are capturing faces, we are found 
different type of problem. These problems are known as blur and noise. Blur and Noise are disturbing the faces and we are not found a clear faces. So we cannot identify the faces. In pre-processing we are improving the faces. We are introducing different algorithms for quality of faces [7]. But they are not clear all the faces. Feature extraction is the third step of processing flow of captured faces. In this step we are identify the each feature of faces. Such as color, pose, eye, nose skin color and mouth etc. These are the basic feature of the faces. We are comparing the entire feature from a relevant faces and the go to next step.

Post- Processing is the fourth step of processing flow of captured images. In this step when we are extract the faces then we are found some blur and noise on the images. Then we are using segmentation and restoration technique to denoise and deblur of images. And finally a clear and resultant faces are found for our research. These resultant faces are using for future research in Biometric Authentication, Surveillance for military services, Human computer interaction, computer vision, pattern analysis, and Multimedia applications.

\section{Related Work on Faces}

Image denoising method is based on the sparse representation of wiener filter. The image modeling and image estimating algorithms is developed in this paper to reduce the noise [8]. There are different type are noise present in the images, Such as Salt noise, Pepper noise, Gaussian noise, Random noise and Hybrid noise. In this paper we are discuss all types of noise and reduce it with the help of wiener filter [9]. In face recognition system, it is very difficult process to capture the image in uncontrolled lighting and moving images. When we are capturing the images, we are found a lot of combining local and high texture based representation. There are different type of illumination, normalization, kernel based feature extraction. And also distance transforms based on matching and multiple feature expression. Working with Local Binary Pattern (LBP) and Local Ternary Pattern (LTP), we have reduced problems from images. The performance of LBP/LTP is totally depending on the face recognition system. The pre-processing method performs pre-processors for an area of data sets, feature sets and lighting conditions. A distance learning transformation is based on the similar features that capture the local figure and geometric reliability of LBP/LTP face images [10]. In this paper we study the hyperspectural image face recognition system. Hyperspectural system is work on the skin color. Facial skin is highlighting the biomedical characteristic of faces. The face detection skin color is combination of chemical component. They have found specific spectral characteristics of color and divine differently for facial face recognition system [11] Image denoising has been very popular technique of learning discriminative model. In image denoising technique, Author are using forwarded step by investigating the construction of feed forward denoising convolution neutral network to improve the performance in very deep architecture. Learning algorithm and residual learning are utilized to enhance the training process and improve the denoising performance. Blind convolution algorithm is reducing the certain level of Gaussian noise with the residual learning strategy. At least learning discriminative approach to tackle with complete image denoising tasks. Such as single image super resolution, JPEG image deblocking and Gaussian noise [12]. It is very challenging task to recognize the face through video and image. We are comparing the dynamic variation of physical structure from dataset. Facial expression is most suitable to recognize the object. Because they are identified that are authenticate or not. This is using both recognition and verification system to extract the value of spatial feature from datasets. These features are helping to introduce the variations of different pose and illumination and help to reduce the differences among identical authentication. In deep evolutional and spatial temporal model is work on extracts the partial whole and dynamic still information. And it is also work on geometry appearance. This model perform effectively and high quality for facial expression recognition [13]. Super and high resolution is an ill posed method. This ill posed problem is aiming to estimate a possible high cost resolution image from a single low cost resolution images. Single image super resolution is patch based method. They are using both internal and external data to learn similar high resolution images. In this paper, there are using wiener filter for image restoration. This approach is working on matching process to the correlate filtering of patch groups in a similarity domain and couple. It is an iterative backprojection framework. The performance of this model is evaluated different number of Single image super resolution bench mark datasets. The proposed algorithm is working on the without using external data. These are work on various scaling factors. Such pose, illumination, brightness, contrast and skin color [14]. After complete the process of face detection, it is important task to register the image in our dataset. Image registration is an important feature of face detector in face recognition system. They are including these method for detecting the face remote sensing, target detection, computer vision, machine learning, blind convolution and image processing. To comparing between two image they are using following parameters. Image content variation, brightness and lighting are very important feature to detect the faces. A multistage registration approach is work on resilient feature, such as point difference, image variation and unconditional lights. A multistage process allowed the utilization of the graph based descriptor. The registration of faces view from pre and post technique provides strong evidence [15]. Faec recognition system have to solve different identification. Suh as pose expression. NFS distance measurement has been embedded in the discriminant anlysis. The repesentation of LLE and LPP , the weighting setting based on the NFS embedding approach is more sysmatic than others. 


\section{Proposed Model for Image Registration}

In figure 2, we are proposed novel technique or model. That is improving the result of Blurred and Noisy Images. Proposed model is work on the blurred and noisy images using digital camera. In this model there are several steps to improving the result. Generally this model is working on the blurred and noisy images. Step 1: First we capture the image with the help of digital camera and biometric sensors. When we are capturing they are not in good quality. And we obtain also stored image in database.

Step2: In this step we obtain filtered image for our experiment.

Step3: In this step we reduce blur and noise. Using Deblur Inference technique and denoise technique. After complete this process, we found deblur and denoise images. Step4: After find deblur and denoise image. We compare this image from database. In this step, we are using surf function for comparing the images. Step5: In this step, we are using random function and found out the each feature of the faces compare with stored in database. If image is found successfully result. Then it will be registered in our database, unless we ignore this image. Because corrupted and ignored resultant images are not using for future work. The capability of the blur parameter identification depends mainly on the relative homogeneity of the blurred image object tracks. The capability of the method is increased when the relative homogeneity of the PSF is higher. If PSF is infinity then identification of capability is very high. Another common source of blur is sinusoidal motion (Vibration).The PSF of high frequency vibration can be determined only by the blur extend. For low frequency technique is vibrate a large variety of point spread function shapes. Can occur, depending on the vibration period in which exposure time is included. Cannon zero crossing identification approach is also performed straight forwardly and separately from the restoration process.

The purpose of this metod, includes very different steps to enhance the effect of the PSF on the blur identification of results.Facial deblur inference technique is used for inferring PSFs using linear model of the variation in facial appearance caused by blur. The equation 1 is used for blur is defined as.

$\mathbf{g}=\mathbf{H f}+\mathbf{n}$

In equation $1 \mathrm{~g}$ is represents the blurred and dirty image called $\mathrm{g}(\mathrm{u}, \mathrm{v})$, matrix $\mathrm{H}$ (Transformation matrix) the PSF, $\mathrm{f}$ is shows the original image and $\mathrm{n}$ is a constant noise. Equation (1) represents a statistical value of image corrupted from blur and noise. $\mathrm{g}$ and $\mathrm{f}$ is including different features of faces recognized from face detector. Image $\mathrm{f}$ can be exactly recovered from the blurred images g. Deblurring by Matching is translated for color image assuming that there are simple transformation that relates the luminance and color. In this technique photograph have been distorted only by shift invariant motion blur on the images indatabase.
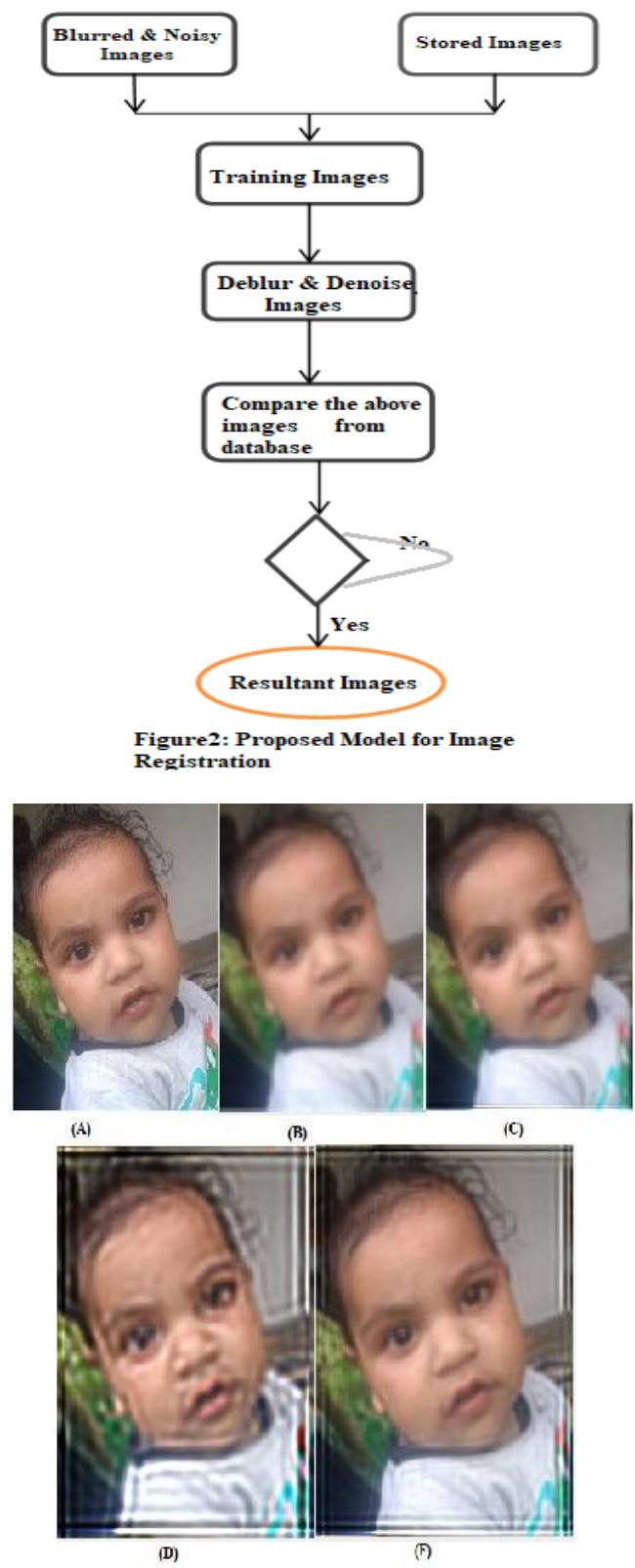

Figure 3: In this figure there are shows different quality of images. (A) Origional Image (B) Blurred Image (C) Deblur Image Undersized PSF (D) Deblur Image Orersized PSF (F) Deblur Image INIT PSF 


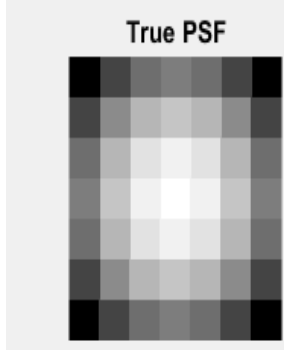

(A)

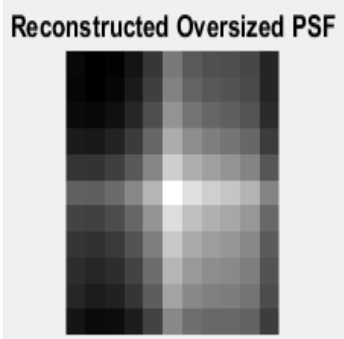

(C)

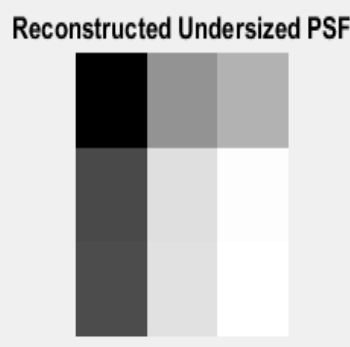

(B)

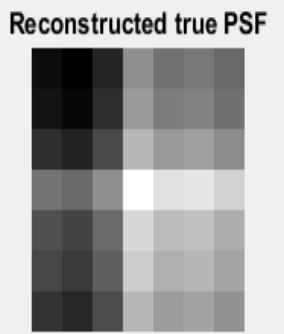

(D)
Figure4: In this figure shows: (A) True PSF (B) Reconstructed Undersized PSF (C) Reconctruted Orersized PSF (D) Reconstructed True PSF

Image Deblurring is using to remove noise and blur from different technique. For spatial-variant kernal, it is possible to locally estimate kernals for different parts of the image and blind deconvolution. That the ability to capture such conditions move into the camera shutter, there by making two shots capture easier and faster. This approach are requiring that the reference image to be sharp. but when additional noise or variation of the lighting conditions alter the considered photograph. The accuracy of the result is highly dependent by the nature of the texture and the size of the common regions shared by the blurred/non blurred photographs.

\section{Result and Analysis}

The experiments are performed on color image (RGB) scale images having natural as well as artificial motion (color, pose. illumination) including blur and noise. The experimental representative images are shown in figure $3 \&$ 4. Figure 3 are shows different improved result of the image. In figure $3 \mathrm{~A}$ is shows original capture and stored image. This is identified pure location of the image. Figure $3 \mathrm{~B}$ is shows the blurred images, which are corrupted using blur. Figure $3 \mathrm{C}$ is shows deblur image undersized of the point spread function. Figure 3D is shows oversized deblur image using point spread function. And last figure $3 \mathrm{~F}$ is shows deblur image INIT point spread function. This image is compared to the original image stored in database. Facial deblur inference model is used for removing the blur from blurred images. This technique is using PSF inference. From equation (1), if the PSF $\mathrm{H}$ and noise $\mathrm{n}$ are constant, an original image $\mathrm{f}$ can be exactly recovered from the blurred image g. This technique is focused on accurately inferring a PSF $\mathrm{H}$ from a blurred facial images g. Figure 4 is shows the contrast and brightness value of reconstructed images. This approach is working on learning feature of facial images and then using proposed model of recognize it in query images of unknown blur. The experimental results with artificial blur and noise are shown in figure. It is observed that proposed novel algorithm has correctly identified the captured and stored image in all restored image from database. The algorithm has performed strongly identified known and unknown in images with including blur and noise. But it has indicated that robust performance in cases of natural motion blur and constant noise. Algorithms for automated restoration of motion blurred images are presented here. This algorithm is depending on different steps. And they are reducing different type of blur and noise including in the images. This algorithm is very important to identify the images. Because in this time for security of everywhere. Point Spread Function is very important feature of the Face recognition. It is likely to have an application in identification tasks in uncontrolled environments. The image restoration algorithms use the knowledge of PSF parameters, blur length and blur direction. Experimental results demonstrate the effectiveness of the algorithms on a wide range of images with natural and varying degree of artificially created motion blur and constant noise.

\section{Conclusion}

In this paper our focus on the behaviour and color of the object. When we are captured the image, then we found dust and noise in our surrounding. Proposed model is helpful to identify the image from our database. And it is useful to the we and our organization. Suppose that face is covered. When This is very difficult to identify the faces. So this algorithm is not identified the faces and they are got this person is dangerous for our environment and life. In feature extraction there are different algorithms. Such as Principal Component Analysis (PCA) and Linear Discriminant Analysis (LDA). These algorithms are only extracting the value of images but our approach is identified image and registered in the database.

\section{References}

[1] A.K. Jain and J.S.Lim, "Handbook of Face Recognition,"Springer,2005.

[2]Masashi Nishiyama, Hidenori Takeshima, Abdenour Hadid, and Jamie Shotton, "Facial Deblur Inference Using Subspace," IEEE Transactions on Pattern Analysis and Machine Intelligence, vol. 33, no. 4, pp. 838-845, April.2011.

[3] J. Ghosn, I.J.Cox. and P.N. Yianilos, Feature Based Image Recognition Using Mixture Distance," In Proceeding of IEEE International Conference on Computer Vision and Pattern Recognition (CVPR'96), San Francisco, CA,USA, pp. 209-216, June 1996. 
[4] J. Jia, "Single Image Motion Deblurring Using Transparency," Proceeding IEEE Conference of Computer Vision and Pattern Recognition, pp. 1-8, 2007.

[5] Zhi-Hua Zhou and Yin Zhang, "Cost-Sensitive Face Recognition," Pattern Analysis and Machine Intelligence, vol. 32, no. 10, pp. 1758-1769, October. 2010.

[6] T. M. Cannon, "Blind Deconvolution of Spatially Invariant Image Blur with Phase," IEEE Transactions on Acoustic Speech and Signal Processing, vol. 24, no. 1, pp.56-63,1976.

[7] D. Hatzinakos and D. Kundur, "Blind Image Deconvolution," IEEE Signal Processing Magazine, vol. 13, no. 3, pp. 43-64, May 1996.

[8] E. Rahtu, T. Ahonen, V. Ojansivu, and J. Heikkila, "Recognition of Blurred Faces Using Local Phase Quantization," Proceeding International Conference of Pattern Recognition, vol. 1, no.1, pp. $1-4,2008$.

[9] Chien-Hsiung, Lee Che-Yen Wen, B.S., "Point spread functions and their applications to forensic image restoration," vol. 1, no.1, pp. 15-26, January 2002.

[10] H. Moon, P.J. Phillips, P.J. Rauss, and S. Rizvi, "The Feret Evaluation Methodology for Face Recognition Algorithms," IEEE Transactions on Pattern Analysis and Machine Intelligence, vol. 22, no. 10, pp. 1090-1104,Oct.2000.

[11] P.J. Flynn, P.J. Phillips, T. Scruggs, K.W. Bowyer, J. Chang, K. Hoffman, J. Marques, J. Min, and W. Worek, "Overview of the Face Recognition Grand Challenge,” Proceeding IEEE Conference of Computer Vision and Pattern Recognition, vol. 1, pp. 947-954, 2005.

[12] J.S.Lim, "Image Restoration by Short Space Spectral Subtraction," IEEE Transactions of Acoustic, Speech and Signal Processing, vol. 28(2), pp. 191-197, 1980.

[13] K.V. Arya, R. Lokhande and P. Gupta, "Identification of Parameters and Restoration Motion Blurred Images," In Proceeding of the 2006 Symposium on Applied Computing (SAC), Dijon, France, vol. 1, no. 1,pp.301-308,April-2006.

[14] Jufu Feng and Yuelong Li, "Automatic Frontal View Face Image Synthesis," IEEE 17th International Conference of Image Processing, vol.12, no.3, pp. 1829-1832.Sep26-29,2010.

[15] N.S. Kopeika and Y.Yitzhaky, "Identification of Blur Parameters from Motion Blurred Images," Graphical Models and Image Processing, vol. 59, no. 5, pp.310-320,1997.

\section{Authors Profile}

Ratnesh Kumar Shukla is currently doing $\mathrm{PhD}$ in Faces Recognition and Face Detection in Image Processing, Department of Computer Science \& Engineering, Dr. APJ Abdul Kalam Technical University (formally Uttar Pradesh Technical University), Lucknow Uttar Pradesh .He obtained her Bachelor of Technology from Uttar Pradesh Technical University Lucknow Uttar Pradesh and his Master's degree in Computer Science and Engineering from Panjab University Chandigarh. His main research work focuses on Face Recognition and Face detection in Image Processing.

Dr. Ajay Agarwal is currently Professor in Department of Computer Science \& Engineering KIET Ghaziabad Uttar Pradesh. He obtained his Bachelor of Technology from I.E.T. Lucknow Uttar Pradesh and Master's in Moti Lal Nehru National institute of Technology (MNNIT) Allahabad, Uttar Pradesh. He also had done PhD from IIT Delhi. He have 30 year above teaching experience in Computer Science \& Engineering.

Dr. Anil Kumar Malviya is currently Professor and Head of Department in Computer Science \& Engineering, Kamla Nehru Institute of Technology Sultanpur. He Obtained Bachelor degree from B.H.U. Banaras and Master's degree from KNIT Sultanpur. He also had done PhD from Dr. B.R.A.U. Agra Uttar Pradesh. He have 23 year above teaching experience in Computer Science \& Engineering, 